\title{
COHERENT STACKING OF SAR IMAGES FOR EFFICIENT INTERFEROMETRIC PROCESSING
}

\author{
Francesco De Zan and Paco López-Dekker \\ DLR (German Aerospace Center), P.O. Box 1116, D-82230 Wessling,Germany.
}

\begin{abstract}
Large datasets of satellite SAR missions dedicated to deformation monitoring will require intensive and dedicated interferometric processing techniques. Decorrelation effects will prevent simple processing approaches, but optimal algorithms are computationally intensive. This paper shows results of a novel efficient algorithm that aims at exploiting scatter component that are stable over time, without being isolated point targets. The concept is proved valid for simulated data and limited examples in X-band and L-band.
\end{abstract}

\section{INTRODUCTION}

Interferometric processing of coherent sets of SAR data has followed in the past several directions. The best results have been obtained either on coherent inteferograms or on selected targets with good quality called PS's[1]. Future missions that aim at a frequent global coverage will make an attempt to map the crustal displacements on the entire world. Large datasets will have to be processed, with arbitrary coherence scenarios and no guarantee for the presence of isolated, point-like targets to be used for phase calibration.

Until a few years ago the situation was complicated by the fact that we had not investigated the theoretical bounds for multi-image SAR interferometry, not even in the simple case of Gaussian scatterer and arbitrary temporal coherence scenario. The situation improved with the derivation of the Cramér-Rao bound (CRB) and the proposal of an optimum estimator [2], assuming only the knowledge of coherence and under the hypothesis of Gaussian scatter.

Now that we know the theoretical bounds we can discuss different estimators. What is proposed in [2] is optimal but requires a considerable computational effort in the case of large datasets. In a paper that will appear shortly [3] we propose a data reduction approach based on the coherent summation of images, before the formation of the interferograms. We show that theoretical results and experiments with TerraSAR$\mathrm{X}$ data confirm the validity of the idea, however better results are obtained with other datasets, for instance in L-band, where we expect to find a scatter component stable over large time spans.

\section{INTERFEROGRAM SELECTION CRITERIA}

As we said, the problem with the optimal maximum-likelihood estimator is that it requires a large computational effort and large datasets will be problematic to handle. In the past several authors have developed euristic strategies to select a reduced number of interferograms. Such strategies are often based on a priori knowledge and coherence. Intuitively, the most straightforward idea is to use the most coherent interferograms, since - taken individually - they are the ones that give the best phases. For example, this is the philosophy of the SBAS technique as presented in [4]. However as it was shown in [2], there are relevant coherence scenarios when this approach can yield large errors (compared to the CRB), especially between the beginning and the end of the phase history.

For a uniform sampling of the time axis and a stationary coherence scenario, the use of lag-1 interferograms is only optimal in the case of purely exponential decorrelation ( $\tau$ is the time constant of the coherence decay):

$$
\gamma(\Delta t)=\exp (-|\Delta t| / \tau)
$$

This can be shown considering that the optimal weighting is given by the inverse of the coherence matrix $\Gamma^{-1}[5,2]$ and such a matrix is tridiagonal if and only if the direct can be expressed as a pure exponential decay.

It is possible to generalize this reasoning to banded matrices. The limitation to a certain time lag $K$ is related to the possibility of modeling the underlying scatter signal as an autoregressive process of order $K$ [6]. Such a modeling is not possible for targets that exhibit a coherence plateau at large temporal baselines

If we wanted to derive a graph from the information contained in the covariance matrix, theoretically one should proceed examining the inverse covariance like it is done for Gaussian graphical models [7].

\section{IMAGE STACKING ALTERNATIVE}

In the specific case of long-term coherence there is an alternative possibility to reduce the number of interferograms. In [3] we suggest to stack coherently subsets of the entire set of images to derive virtual images. 
For example we build the virtual image $v_{1}$ from a subset of the images $y_{n}$ :

$$
v_{1}(l)=(1 / S) \sum_{n \in S_{1}}^{S} y_{n}(l) \exp \left(-j \phi_{n}^{1}\right)
$$

Here $y_{n}(l)$ represent the complex value of pixel $l$ in image $n$. The set $S_{1}$ is a subset of the dataset positioned at the beginning, temporally speaking. The problem of maintaining coherence between the images is solved by correcting (rephasing) with the $\phi_{n}^{1}$ 's, which are the interferometric phases of each image with respect to a reference in the subset $S_{1}$. These phases can be estimated for example with the algorithm in [2], limited to the subset.

The same stacking operation can be repeated for a different subset positioned at the end of the dataset. Finally one can build the interferogram between the two virtual images and multilook it:

$$
I_{1,2}=\sum_{l=1}^{L} v_{1}(l) v_{2}^{*}(l) .
$$

This equation contains implicitely all the interferograms between the images in the first subset $S_{1}$ and in $S_{2}$ and allows to make interferometric measures spanning a large time interval.

\section{SIMULATIONS}

We simulated complex-gaussian data according to the following coherence law (for $n \neq m$ ):

$$
\gamma_{n, m}=\left(\gamma_{0}-\gamma_{\infty}\right) \exp (-|n-m| / \tau)+\gamma_{\infty} .
$$

It represents an exponential decay from $\gamma_{0}$ to $\gamma_{\infty} \neq 0$ with a time constant $\tau$. We assume a uniform sampling of the temporal axis. We performed simulations with 200 images from which two subsets of 30 scenes are taken, and where the coherence decays from 0.8 to 0.2 with a time constant three times the repeat-pass time.

In this case the loss on the variance of the phase difference between the first and the last image in the stack $\left(\phi_{N}-\phi_{1}\right)$ is limited to $0.6 \mathrm{~dB}$ [3]. This has to be compared with something like 5-7 $\mathrm{dB}[8]$ when one uses only the most coherent interferograms (between one image and the next in the time series).

\section{EXPERIMENTS WITH TERRASAR-X}

We apply the proposed procedure to a stack of 30 TerraSAR$\mathrm{X}$ images acquired between 2008 and 2009 in the region of Crotone, Italy. The images have been coregistered to a common reference and a topographic phase component has been removed (SRTM). Two subsets have been selected, one comprising 15 and the other 8 images.
The synthesis of the two coherent images has been done according to (2) after having estimated the phases for each subset with the algorithm found in [2] and the coherence magnitudes from the data themselves. The coherence between the virtual images is about 0.9 on the airport runway (Fig. 1, right), which is a value not attained by any pair between the two subsets. The best coherences between the two subsets are around 0.6, and only in a few interferograms. Fig. 1 (right) shows the phase reconstructed with the proposed procedure.

\section{EXPERIMENTS WITH ALOS-PALSAR}

The proposed coherent stacking can be useful if some components of a distributed scatterer exhibit long-term coherence. This approach could be interesting for relatively long wavelength SAR missions (L-band) with short repat-pass time, if some kind of smart interferometric processing is required due to the large amount of acquired data.

The described procedure is applied to a stack of $7+6 \mathrm{SAR}$ images from the ALOS sensor PALSAR over Mt. Etna, Italy. Since some acquisitions are dual-pol and others are singlepol, only the common polarimetric channel is considered (HH). Special care has to be taken since the bandwidth is also different between acquisitions.

The resulting phase for a small patch of the images is shown in Fig. 2. For comparison we show the multilooked phase of the direct interferogram. The figure on the right is obtained with a variant of the proposed algorithm (see [3]), where the weights in the coherent combination of the images are chosen to maximize the coherence. As expected, the Lband data seem to comply more extensively to the long-term coherence hypothesis and the results are more encouraging.

The proposed algorithm is only suited to a specific coherence scenario, thus for a systematic interferometric processing one could adopt a dual approach. On one side one would do safely process the most coherent interferograms, on the other one would attempt to retrieve the long term coherent component, in order to achieve the best performance.

\section{AKNOWLEDGMENTS}

We would like to acknowledge the Science Coordinator for TerraSAR-X and JAXA for the data used in this paper and Dr. Pau Prats for the interferometric routines.

\section{REFERENCES}

[1] A. Ferretti, C. Prati, and F. Rocca, "Permanent scatterers in SAR interferometry," Geoscience and Remote Sensing, IEEE Transactions on, vol. 39, no. 1, pp. 8-20, Jan 2001.

[2] A. Monti Guarnieri and S. Tebaldini, "On the exploitation of target statistics for SAR interferometry applications," 

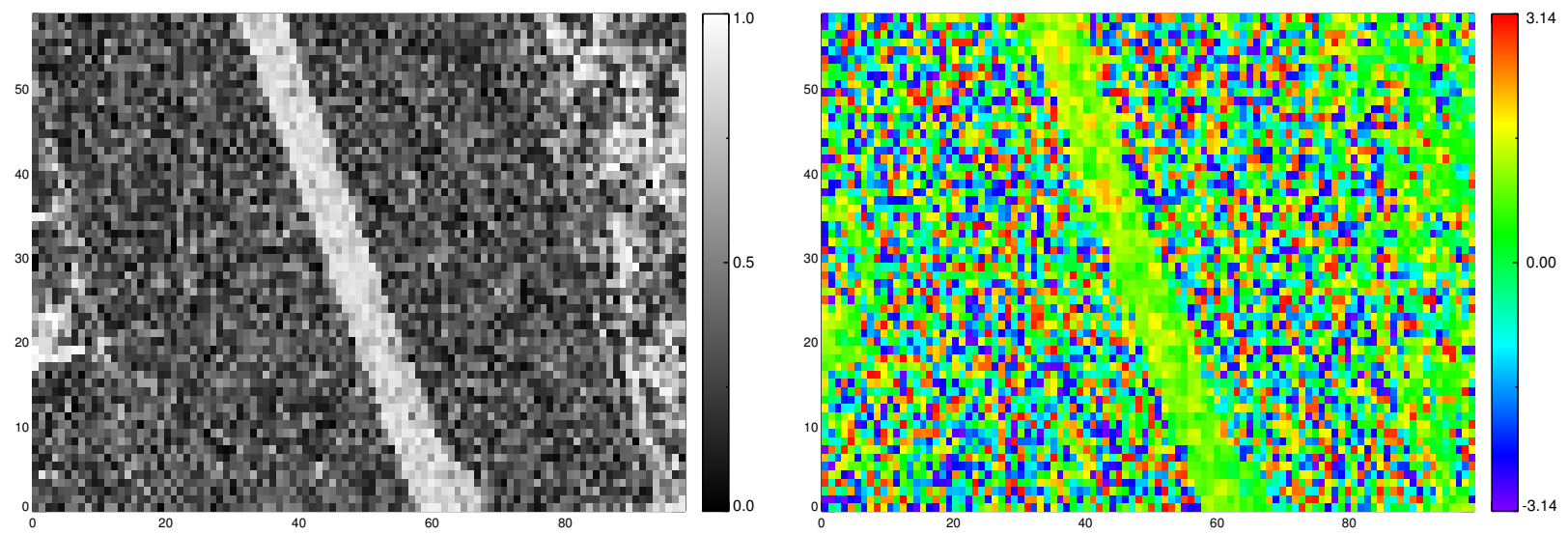

Fig. 1. Left: Coherence between virtual images. The coherence is computed on $5 \times 5$ windows, whereas the phases for the synthesis are computed on 10x10 windows. Right: The interferometric phases between two images (2008-06-08 and 2009-1221 ), estimated with the proposed procedure, on $5 \times 5$ pixel windows.
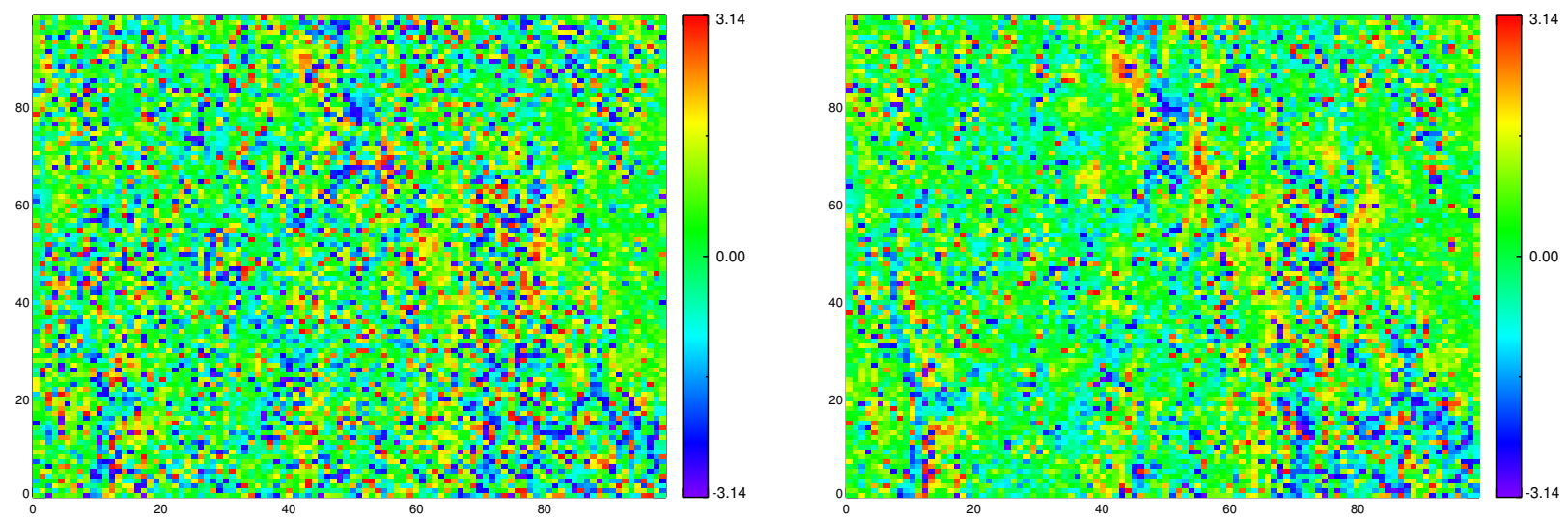

Fig. 2. Left: the interferometric phases between two images (2007-10-30 and 2009-12-20), estimated with the proposed optimized procedure, on 10x10 pixel windows. Right: the same interferometric phases estimated with the proposed algorithm.

IEEE Trans. Geosci. Remote Sensing, vol. 46, pp. 34363443, Nov 2008.

[3] F. De Zan and P. López-Dekker, "SAR image stacking for the exploitation of long-term coherent targets," Geoscience and Remote Sensing Letters, IEEE, 2011.

[4] R. Lanari et al., "An overview of the Small BAseline Subset algorithm: a DInSAR technique for surface deformation analysis," Pure and Applied Geophysics, vol. 164, no. 4, pp. 637-661, Apr 2007.

[5] A. Ferretti, F. Novali, F. De Zan, C. Prati, and F. Rocca, "Moving from PS to slowly decorrelating targets: A prospective view," Proc. of EUSAR 2008, 2008.
[6] A. Kavčić and J. Moura, "Matrices with banded inverses: inversion algorithms and factorization of Gauss-Markov processes," Information Theory, IEEE transactions on, vol. 46, no. 4, pp. 1495-1509, Jul 2000.

[7] A. Wiesel et al., "Covariance estimation in decomposable Gaussian graphical models," Signal Processing, IEEE Transactions on, vol. 58, no. 3, pp. 1482-1491, Mar. 2010.

[8] F. De Zan, P. Prats, and G. Krieger, "Mission design and peformance for systematic deformation measurements with a spaceborne SAR system," IGARSS 2009, Proceedings of, Nov 2009. 